\title{
A 15 GeV Electron Accelerator for Europe
}

J. Arvieux of the Laboratoire National Saturne, Saclay, France, describes a new European facility for nuclear physics.

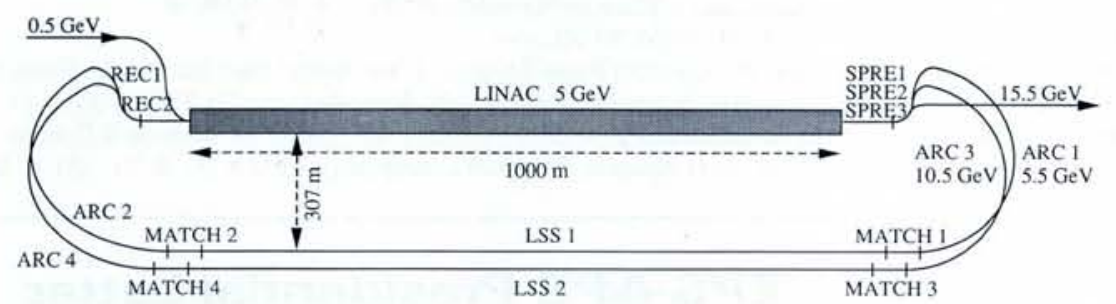

Schematic layout of the three-pass recirculating LINAC being proposed as a future 15-20 GeV electron accelerator for nuclear physics. Two, long, straight sections (LSS) are matched by matching sections (MATCH) to arc sections coupled to recombination (REC) and spreader (SPRE) sections that lead to and from a $1 \mathrm{~km}$ long LINAC.

The project for a $15-30 \mathrm{GeV}$ European Electron Accelerator is gaining momentum after the publication in 1991 of the Nuclear Physics European Collaboration Committee (NuPECC) report Nuclear Physics in Europe: Opportunities and Perspectives on future facilities and instrumentation (see also EN 21 (1990) 111). The report assessed six sub-fields where Europe plays an important, if not dominant, rôle. Among the recommendations, the one concerned with studies using high energy electron probes stand out by its clarity: "NUPECC recommends that a major initiative be launched now to develop a proposal for a European CW (continuous wave) electron accelerator in the $15 \mathrm{GeV}$ region".

The physics case of such a facility has been discussed at numerous conferences and workshops [Seillac (1988), Dourdan (1990), Amsterdam 1991), Penn State, USA (1992)]. Summarising in a few words, its goal will be to study QCD in its perturbative (including non-leading order effects) and non-perturbative aspects. One hopes to understand the basic and opposing phenomena of confinement (when quarks stick together in a physical hadron) and hadronization (when quarks escape from it). In particular, one would like to confirm colour transparency where due to the uncertainty principle only small size quark configurations can be excited in a nucleus at large momentum transfer. These configurations have a lifetime of the order of 1 fermi per $(\mathrm{GeV} / \mathrm{c})^{2}$ transferred so they evolve back to normal hadrons after only a few fermis. Using a nucleus as a laboratory whose size, mass and density can be adjusted is one, and maybe the only, way to characterise them.

The choice of an initial energy of $15 \mathrm{GeV}$ allows the study of charm electroproduction ( $\mathrm{J} / \mathrm{psi}$ and associated charm) at sizable cross-sections - a regime of special interest since the large mass of charmed hadrons allows detailed QCD predictions. Studies of $\mathrm{J} /$ psi production in nuclei will also permit one to explore the evolu- tion of $c \bar{c}$ pairs in the nuclear medium and to help to clarify quark-gluon plasma formation in nucleus-nucleus collisions. A fairly complete overview of the physics which could be done with the new facility has been given in Europhysics News 21 (1990) 213.

\section{European Steering Committee}

Following the recommendations of a preliminary NuPECC report in July 1991, an European Steering Committee (ESC) chaired by $\mathrm{T}$. Walcher from Mainz has been set up by funding agencies in France, Germany, Italy, and The Netherlands. As stated by NuPECC, the ESC aims to:

- identify the key experiments;

- determine the initial energy and propose an optimum accelerator design;

- establish the instrumentation required for the initial experimental programme;

- submit a project report to funding agencies by the end of 1992 .

To fulfill this ambitious task the ESC has organised working structures to develop the scientific and technical aspects of the proposal. They are:

- a Scientific Advisory Committee (SAC) comprising eminent nuclear and particle physicists which will assess the scientific merits of the project;

- calls for proposals have been sent to physicists who have expressed interest in the new facility. Proposals may range from rather general expressions of interest dealing with conceptual or theoretical ideas to full experimental proposals, including a detailed description of the necessary apparatus; - a workshop to be organised in Mainz (7-9 October 1992) to discuss proposals and technical aspects of the machine. In the meantime, topical workshops and working sessions will be organised in different European countries to help the elaboration of proposals and to promote cooperation on an European scale.

European Electron Machine Committee

Concerning the machine design, an European Electron Machine Committee has been set up. After a fairly exhaustive review of different possible designs, studies are focussing on a rather powerful LINAC $(5 \mathrm{GeV})$ with only two recirculations (a three-pass LINAC), as shown in the figure. The specifications for the superconducting RF cavities call for: a frequency in the $1.3-1.5 \mathrm{GHz}$ range (corresponding to the frequency particle bursts are separated by less than a nanosecond and the detectors see essentially a DC structure); a field gradient of $10 \mathrm{MV} / \mathrm{m}$; an intensity of $>10 \mu \mathrm{A}$ per circulating beam (20 $\mu \mathrm{A}$ seems to pose no problems even for nine-cell cavities presently being studied for the TESLA project in the context of a future linear collider for particle physics [see EN 22 (1991) 184], but higher intensities may require tailored designs); a filling factor (ratio of overall length to active length) of 2; and a cost of the order of $20 \mathrm{kSFR} / \mathrm{MeV}$. Although ambitious, these characteristics remain realistic thanks to the outstanding progress made in several European organizations in developing and fabricating superconducting RF cavities.

The energy resolution has been chosen to be of the order of $\Delta E / E \approx 3 \times 10^{-4}$ FWHM at $15 \mathrm{GeV}$. The machine is designed in such a way that an eventual upgrade to $30 \mathrm{GeV}$ will not require the construction of a new machine but only upgrading of the RF system and the magnet power supplies. Since the energy resolution is essentially determined by synchrotron radiation losses in the recirculating arcs, the size of the machine is approximately the same for 15 and $30 \mathrm{GeV}$ if one accepts a limitation of the energy resolution of $10^{-3}$ at $30 \mathrm{GeV}$ (which would still allow meson production to be separated from inelastic nuclear reactions).

The success of the project will largely depend on the commitment of a sizeable part of the European nuclear physics community. All contributions are welcome and the persons interested in receiving further information should contact the Chairman or a member of the ESC (see below).

\section{European Electron Accelerator Project EUROPEAN STEERING COMMITTEE}

T. Walcher (Chairman), Inst. f. Kernphys., Postfach 3980, W-6500 Mainz 1 +49 (6131) 395196 / 3929 64; walcher @ vkpmza.kph.uni-mainz

J. Arvieux, Lab. Nat. Saturne, CEN Saclay, F-91191 Gif-sur-Yvette Cédex +33 (1) $69082203 / 69082970$; arvieux@ frcpn11

E. de Sanctis. Lab. Naz. di Frascati, CP 13, I-00044 Frascati +39 (6) $9403316 / 94035$ 59; desanctis@irmlnf

P. de Witt-Huberts, NIKHEF-K, Postbus 41 882, NL-1009 DB Amsterdam +31(20) 5922163 /592 2165 ; marijke@ nikhefk.nikhef.nl 